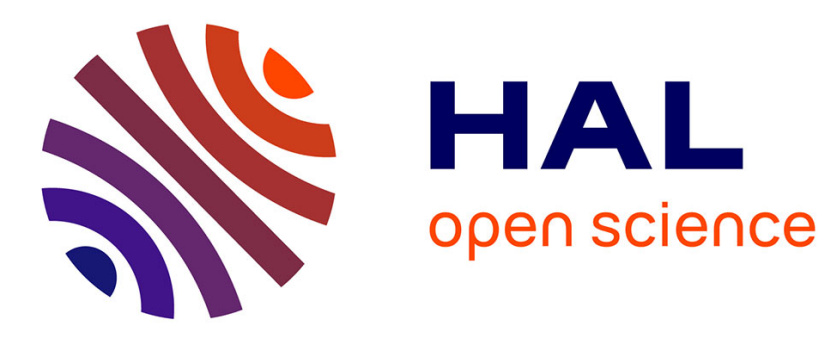

\title{
La fin de la vie et le droit européen
}

Estelle Brosset

\section{To cite this version:}

Estelle Brosset. La fin de la vie et le droit européen. Negri (S.). Between Life and Death: Regulating Advance Directives. National and International Perspectives, Martinus Nijhoff Publishers, pp. 87-106, 2011. hal-00872015

\section{HAL Id: hal-00872015 https://hal.science/hal-00872015}

Submitted on 11 Oct 2013

HAL is a multi-disciplinary open access archive for the deposit and dissemination of scientific research documents, whether they are published or not. The documents may come from teaching and research institutions in France or abroad, or from public or private research centers.
L'archive ouverte pluridisciplinaire HAL, est destinée au dépôt et à la diffusion de documents scientifiques de niveau recherche, publiés ou non, émanant des établissements d'enseignement et de recherche français ou étrangers, des laboratoires publics ou privés. 


\title{
La fin de la vie et le droit européen
}

\author{
Estelle Brosset ${ }^{1}$, \\ «La vie c'est ça, un bout de lumière qui finit dans la nuit» \\ Louis- Ferdinand Céline, Voyage au bout de la nuit.
}

Lumière qui devient nuit, la vie prend inexorablement fin, suivant ici un phénomène ancestral, universel et naturel, mais néanmoins tragique ${ }^{2} \ldots$

... Dans cette nuit, trouve-t-on la figure du droit européen ? La réponse n'est pas aisée, mais nécessaire si l'on veut avoir une vue exacte, ce qui est l'ambitieux objectif de cet ouvrage, du droit positif en la matière.

Elle n'est pas aisée parce l'existence d'un droit européen sur des questions aussi intimes et complexes que celle de la fin de vie pose tout à la fois des questions relatives à la légitimité et la faisabilité d'un droit européen. L'universalisation, plus particulièrement l'européanisation est-elle possible, surtout au regard des conflits profonds, d'ordre spirituel, culturel ou idéologique, qui divisent les États sur la question de la vie ? La renationalisation ${ }^{3}$ c'est-à-dire le maintien d'une diversité juridique n'est-elle pas inévitable? Le consensus, même exclusivement européen, est-il possible sur ces questions? Il faut d'emblée dire que le consensus n'est pas facilité par la profusion des concepts - euthanasie active ou passive, suicide assisté, soins palliatifs, stade terminal, non acharnement thérapeutique...- et la variabilité des définitions. À l'échelle parfois d'un seul pays, la question du vocabulaire employé pour décrire la fin de vie ou encore à ce qu'on appelle la phase terminale suscite débat ${ }^{4}$. Déjà en 1976, l'Assemblée Parlementaire du Conseil de l'Europe dans une Résolution 613 « craignant que l'incertitude quant aux critères les plus valables de définition de la mort ne soit un tourment inutile » (...) invitait « à examiner, à la lumière des connaissances et techniques médicales actuelles, les critères en vigueur dans les différents pays européens pour

\footnotetext{
${ }^{1}$ Maître de conférences habilité à diriger les recherches en droit public,Université Paul Cézanne (France), membre du Centre de droit de la santé, membre associé au Centre d'Etudes et e Recherches Internationales et Communautaires, estelle.brosset@univ-cezanne.fr.

${ }^{2}$ «D'un côté la mort reste bien un phénomène naturel, qui est dans l'ordre des choses, qui permet que les vivants meurent pour que d'autres renaissent; d'un autre côté, la mort est une épreuve qui fait disparaître un être unique insubstituable, ce qui reste un scandale pour la conscience et un mystère pour l'esprit » : CDBI, 35 ième réunion, 2-5 décembre 2008, Les décisions médicales dans les situations de fin de vie et les implications éthiques des choix possibles, rapport préparé par le Dr Lucie Hacpille, http://www.coe.int/t/dg3/healthbioethic/Activities/09_Euthanasia/default_fr.asp, p. 13.

3 Voir Catherine Girard, «Le droit international de la bioéthique, 1'universalisation à visage humain ?» in Stéphanie Hennette-Vauchez (dir.), Bioéthique, biodroit, biopolitique, réflexions à l'occasion de la loi du 4 août 2004, LGDJ, 2006, p. 51 et s.

${ }^{4}$ Voir l'effort de clarification en France réalisé par le Rapport de l'Assemblée nationale sur l'accompagnement de la fin de vie, M. Jean Léonetti, n 1708, 30 juin 2004, pt 1-1.
} 
constater le décès, et à formuler des propositions d'harmonisation de ces critères qui permettent d'en généraliser l'application non seulement dans les hôpitaux, mais dans toute la pratique médicale ». Surtout s'il est un domaine où l' «indécidabilité »5 est forte, c'est celui de la mort, indécision personnelle quant aux choix à opérer pour soi, pour ses proches, indécision encore plus grande au plan collectif quand aux normes à consacrer. "Même si, à certains égards, on assiste à un certain rapprochement entre pays européens concernant l'éthos démocratique, les valeurs communes, on constate aussi des différences sensibles quant à l'approche de certains problèmes de société tels que l' euthanasie, l'aide aux mourants ou les soins palliatifs. Sur ces sujets comme dans d'autres domaines, l'Europe institutionnelle est une chose, celle des pratiques culturelles, des choix de valeurs en est une autre. Et d'un pays à l'autre, l'écart entre le discours et la réalité peut s'avérer considérable $»^{6}$.

Il n'en demeure pas moins que l'on peut repérer, ici ou là, une «intrusion ${ }^{7} \mathrm{du}$ droit européen. Il faut dire que plusieurs facteurs y ont participé, principalement, les changements radicaux qu'a connu la fin de la vie. La technologie médicale a en effet, ces dernières décennies, non seulement permis l'allongement de l'espérance de vie mais aussi prolongé le temps d'incertitude entre la vie et la mort, parfois aux prix d'interminables agonies. La fin de vie est désormais de plus en plus médicalisée et hospitalière et la mort perçue comme un échec -«la mort c'est quand la médecine décide d'arrêter $»^{8-}$, échec que l'on s'efforce nécessairement de refouler dans une sorte de syndrome de «déni de la mort ${ }^{9}$. Logiquement, cette transformation a suscité de nouvelles interrogations, en particulier relatives aux droits fondamentaux de la personne et a débouché sur des adaptations législatives parfois très variables en fonction des Etats. Or, le droit européen, en ce qu'il consacre un corpus de droits fondamentaux (le droit à a vie ou la protection de la vie privée) et en ce qu'il permet l'harmonisation des législations $^{10}$, ne pouvait manquer d'interférer avec ce débat.

Il n'est dès lors pas surprenant de recenser quelques recommandations de l'Assemblée Parlementaire du Conseil de l'Europe ${ }^{11}$, quelques rapports du Comité

\footnotetext{
5 Jean-Luc Baudoin et Catherine Labrusse-Riou, Produire l'homme : de quel droit ?, PUF, Paris, 1987, p. 260.

${ }^{6}$ Philippe Pedrot, Existe-t-il un droit de mourir dans la dignité ? RDSS, 2002, p. 477.

${ }^{7}$ Louis Dubouis, La fin de vie et le droit européen in Antoine Leca et Sophie De Cacqueray, La fin de vie et l'euthanasie, Les études hospitalières, 2008, p. 77.

${ }^{8}$ CDBI, 35 ième réunion, 2-5 décembre 2008, Les décisions médicales dans les situations de fin de vie et les implications éthiques des choix possibles, rapport préparé par le Dr Lucie Hacpille, http://www.coe.int/t/dg3/healthbioethic/Activities/09_Euthanasia/default_fr.asp, p. 3.

${ }^{9}$ Rapport de l'Assemblée nationale sur l'accompagnement de la fin de vie, M. Jean Léonetti, ${ }^{\circ} 1708,30$ juin 2004, pt 1-1.

${ }^{10}$ L'harmonisation est souvent «demandée » par l'opinion publique qui comprend mal les disparités entre législations. "Que l'euthanasie soit interdite à Lyon et Lille mais qu'on puisse y recourir si l'on se rend à Zurich ou Bruxelles provoque un réel malaise » : Louis Dubouis, La fin de vie et le droit européen op.cit., p. 78. ${ }^{11}$ Recommandation 1418 (1999), Protection des droits de l'homme et de la dignité des malades incurables et des mourants ; Recommandation 779 (1976) relative aux droits des malades et des mourants.
} 
Directeur pour la bioéthique ${ }^{12}$, quelques articles éparses dans la Convention d'Oveido ${ }^{13}$ ou dans la Charte des droits fondamentaux de l'Union européenne ${ }^{14}$. Constatons toutefois que, malgré quelques exceptions, comme la Convention de biomédecine, qui cependant comporte le plus souvent des formulations extrêmement prudentes, la portion la plus importante du droit européen en la matière est constituée de rapports et études de comités d'éthique, de déclarations des États ou encore de résolutions des organisations compétentes, en bref, d'un ensemble juridique non contraignant parfois qualifié d' «infra droit» ${ }^{15}$. La redondance est d'ailleurs telle qu'il faut nécessairement y voir «plus qu'une maladie de jeunesse » ${ }^{16}$, les questions de bioéthique comme celles de la fin de vie s'inscrivant plus facilement "dans l'argile des résolutions dont la force juridique est faible que dans l'airain de conventions contraignantes ${ }^{17}$. À ces textes s'ajoute toutefois la jurisprudence, en particulier la jurisprudence de la Cour européenne des droits de l'homme, qui a le mérite de dégager, à la charge des Etats, de véritables obligations, limitées toutefois aux cas d'espèce. Concernant la fin de vie, la jurisprudence est maigre, mais formée d'un arrêt resté «dans les annales » ${ }^{18}$, l'arrêt Pretty ${ }^{19}$. On se souvient que Mme Pretty, la requérante, âgée de 43 ans, atteinte d'une maladie neurodégénérative, irréversible et incurable ${ }^{20}$, mais qui gardait toutes ses facultés intellectuelles, souhaitait que son mari soit autorisé à l'assister dans son suicide sans encourir de poursuites. Elle affirmait devant la Cour que le refus par le Director of Public prosecutions d'accorder une indemnité de poursuites à son mari à la suite d'une aide au suicide et la prohibition de cette aide édictée par le droit anglais ${ }^{21}$ enfreignaient les

\footnotetext{
${ }^{12}$ Rapport sur les lois en vigueur ou pratiques appliquées dans les Etats membres relatives aux questions soulevées par la Recommandation 1418 (1999) de l'Assemblée parlementaire relative à la protection des droits de l'homme et de la dignité des malades incurables et des mourants [831 Réunion] : Réponses au questionnaire pour les Etats membres relatif à l'euthanasie, 20 janvier 2003, CDBI/INF (2003) 8

${ }^{13}$ Convention signée sous l'égide du Conseil de l'Europe à Oviedo le 4 avril 1997. Voir notamment l'article 1 : «Les Parties à la présente Convention protègent l'être humain dans sa dignité et son identité et garantissent à toute personne, sans discrimination, le respect de son intégrité et de ses autres droits et libertés fondamentales à l'égard des applications de la biologie et de la médecine. Chaque Partie prend dans son droit interne les mesures nécessaires pour donner effet aux dispositions de la présente Convention ». Voir également l'article 5 ou l'article 9 voir plus bas.

${ }^{14}$ Article 3 :

«1.Toute personne a droit à son intégrité physique et morale.

2. Dans le cadre de la médecine et de la biologie, doivent notamment être respectés :

- le consentement libre et éclairé de la personne concernée, selon les modalités définies par la loi,

- l'interdiction des pratiques eugéniques, notamment celles qui ont pour but la sélection des personnes,

- l'interdiction de faire du corps humain et de ses parties, en tant que tels, une source de profit,

- l'interdiction du clonage reproductif des êtres humains ».

15 L'expression est tirée de l'article particulièrement éclairant de Sandrine Maljean-Dubois, « Bioéthique et droit international », Annuaire Français de Droit International, 2000, p. 86.

16 Sandrine Maljean-Dubois, «Bioéthique et droit international », op. cit., p. 110.

17 Noëlle Lenoir et Bertrand Mathieu, Les normes internationales de bioéthique, PUF, 1998. p. 43.

${ }^{18}$ Jean-Pierre Marguénaud, Hymnes à la vie et à l'autonomie personnelle, RTD Civ. 2002 p. 858.

${ }^{19}$ Cour EDH 4e sect. 29 avr. 2002, Pretty c/Royaume-Uni, Rev. science crim.2002.645, note F. Massias ; D.2002.1596 ; RJPF 2002, 7-8/11, p. 11 obs. E. Garaud ; JCP 2002.I.157, n 1 et 13, obs. F. Sudre ; Defrénois, 2002.1131, obs. Ph. Malaurie.

${ }^{20}$ Une sclérose latérale amyotrophyque qui l'a paralysait du cou jusqu'aux pieds.

${ }^{21}$ Le suicide en droit anglais, n'est pas considéré comme une infraction mais Mme Pretty était dans l'impossibilité d'accomplir un tel acte sans assistance. Or, aider quelqu'un à mettre fin à ses jours est en revanche, sanctionné par l'article $2 \S 1$ er de la loi de 1961 sur le suicide.
} 
droits garantis par la Convention européenne, en particulier les articles 2, 3, 8, 9 et 14 . Rejetant la demande de Mme Pretty et estimant que la Grande-Bretagne n'avait violé aucun des articles de la Convention, les juges ont, dans cette affaire, fixé plusieurs jalons importants s'agissant de la confrontation entre les droit garantis par la Convention et la décision de mettre fin à sa vie.

Ce corpus de droit européen peut être appréhendé au travers d'un double mouvement: si d'une part l'on assiste à la protection par le droit européen de l'autonomie des individus désireux de «bien » finir leur vie (I), d'autre part, le droit européen reste relativement en retrait, discret lorsque l'individu décide de mourir, ne se prononçant pas ou très peu dans le débat sur l'euthanasie (II).

\section{I- BIEN FINIR SA VIE}

La fin de la vie a été profondément modifiée non seulement par les avancées importantes des technologies de maintien de la vie et d'amélioration de la qualité de la fin de vie mais encore par la place accordée à l'autonomie du patient dans l'adoption des décisions médicales le concernant. Le droit européen a incontestablement participé à la consolidation de cette autonomie, affirmant d'abord, d'une manière peu contraignante, le droit de refuser un traitement médical non désiré, y compris si une telle décision le mène à la mort $(\mathrm{A})$, consacrant ensuite une véritable obligation pour les Etats de respecter le droit de chaque personne de choisir sa mort (B).

\section{A. L'affirmation d'un droit de refuser un traitement médical non désiré}

Le consentement au traitement médical et le droit subséquent d'un individu de refuser un traitement médical non désiré, même si ce traitement est vital pour lui, ont été reconnus de longue date en droit européen. L'accumulation d'affirmations est cependant tempérée par la faible portée contraignante de celles-ci.

\section{1- L'accumulation de références}

L'accumulation est nette. La Recommandation 779 du 29 janvier 1976, de l'Assemblée parlementaire du Conseil de l'Europe, relative aux droits des malades et des mourants précisait déjà que les médecins doivent avant tout respecter la volonté de l'intéressé en ce qui concerne le traitement ${ }^{22}$. La Déclaration sur la promotion des droits des patients en Europe de 1994 consacre également le consentement en disposant qu'aucun acte médical ne peut être pratiqué sans le consentement éclairé et préalable du patient, et ajoute que ce dernier a le droit de refuser un acte médical ou de l'interrompre en exigeant du médecin une claire explication de la portée d'un tel choix ${ }^{23}$. La Convention sur les droits de l'homme et la biomédecine dispose dans son article 5

$22 \S 4$ de ladite Recommandation.

${ }^{23} \S 3.1$ de ladite Déclaration. 
qu'une intervention dans le domaine de la santé ne peut être effectuée qu'après avoir obtenu le consentement libre et éclairé de la personne concernée et que, ce qui est une première en droit international, «la personne concernée peut, à tout moment librement retirer son consentement». Enfin, l'on peut citer également la Charte des droits fondamentaux de l'Union européenne du 18 décembre 2000, qui consacre de manière identique le droit des citoyens à décider librement sur les questions médicales après en avoir été informés. Naturellement, ce droit ne vaut que si le patient est conscient et capable d'exprimer sa volonté ou s'il ne l'est plus, s'il a exprimé sa volonté à un moment où il était pleinement capable de le faire à travers une directive anticipée. Plusieurs instruments évoquent d'ailleurs cette hypothèse. La Convention de biomédecine précise, dans son article 9, que les souhaits manifestés avant l'intervention médicale par un patient qui ne se trouve pas en situation d'exprimer sa volonté au moment de cette intervention, doivent être entendus car ils font partie du consentement nécessaire. Dans le prolongement, la Recommandation 1418 du 25 juin 1999 de l'Assemblée parlementaire enjoint expressément les Etats membres de tout mettre en oeuvre pour faire « respecter les instructions ou la déclaration formelle (living will) rejetant certains traitements médicaux données ou faites par avance par des malades incurables ou des mourants désormais incapables d'exprimer leur volonté $»^{24}$.

2- De faibles contraintes pour les Etats

Précoces et continues, ces affirmations n'en demeurent pas moins faiblement contraignantes. D'abord, la majorité des textes n'est pas dotée d'une force obligatoire et n'oblige en rien juridiquement les Etats qui sont plutôt « invités à ». Certes, il y a bien la Charte des droits fondamentaux, mais sa portée contraignante est relativement récente ${ }^{25}$. Il y a bien également la Convention biomédecine. Toutefois, cet instrument contraignant souffre de plusieurs défauts. D'abord, elle n'a recueilli qu'un faible nombre de ratifications ${ }^{26}$; ensuite, elle a établit un mécanisme de contrôle du respect de ses dispositions «particulièrement timide ${ }^{27}$; enfin, ses formulations restent le plus souvent très prudentes. Il en est ainsi de l'article 9 de la Convention relative aux directives anticipées ${ }^{28}$. Ladite disposition n'oblige pas en effet les Etats à conférer à ces documents un caractère contraignant : «les souhaits précédemment exprimés (...) seront pris en compte». D’ailleurs le rapport explicatif dit les choses sans détours : «la prise en compte (...) ne signifie pas que ceux-ci devront être nécessairement suivis. Ainsi, par

\footnotetext{
${ }^{24} \S 9$, b, IV de ladite Recommandation.

${ }^{25}$ Elle a été acquise dans le traité de Lisbonne entré en vigueur le $1^{\text {er }}$ décembre 2009.

${ }^{26}$ La Convention, entrée en vigueur le $1^{\text {er }}$ décembre 1999, n'a obtenu, pour l'heure, que 26 ratifications.

${ }^{27}$ Christian Byk, La Convention européenne sur la biomédecine et les droits de l'homme et l'ordre juridique international, JDI, 1, 2001, p. 57. Voir notamment l'article 29 de la Convention : la Cour européenne des droits de l'homme ne se voit pas conférer de compétence similaire à celle qu'elle exerce dans le cadre de la Convention européenne des droits de l'homme, son intervention demeurant simplement consultative et limitée puisqu'elle devra être dégagée de tout lien avec un litige concret soumis à une juridiction nationale.

${ }^{28}$ À noter toutefois que les directives anticipées incluent non seulement les testaments de vie qui expriment les préférences des personnes concernant les recours à des traitements précis et la procuration pour soins de santé qui permet à une personne de désigner quelqu'un pour prendre des décisions en matière de santé en leur nom.
} 
exemple, lorsque ces souhaits ont été exprimés très longtemps avant l'intervention et que les conditions scientifiques ont évolué, il peut être justifié de ne pas suivre l'opinion du patient. Le praticien doit donc, dans la mesure du possible, s'assurer que les souhaits du patient s'appliquent à la situation présente et sont toujours valables, compte tenu notamment de l'évolution des techniques médicales ${ }^{29}$. Autre prudence, l'article ne prévoit pas les conditions, notamment formelles, d'élaboration et donc de validité de ces documents, laissant en l'espèce une marge d'appréciation aux Etats. Le rapport du CDBI sur les souhaits précédemment exprimés au sujet des soins de santé30 évoque l'hypothèse de l'adoption de dispositions européennes plus précises qui pourraient être incluses dans un protocole additionnel, réglant non seulement les exigences formelles mais aussi l'effet juridique de ces directives anticipées. Cependant, au vu de la forte disparité des statuts juridiques des directives anticipées dans les pays européens, recensés dans le même rapport, le consensus paraît hypothétique.

D’ailleurs, la réalité des pratiques médicales est bien souvent en décalage avec ce principe d'autonomie ainsi qu'en attestent plusieurs études visant à récolter des données européennes. Une étude, étude Ethicus, s'est déroulée entre 1999 et 2000 dans 37 services de réanimation de 17 pays européens. Ayant pour objet d'examiner les décisions de fin de vie en réanimation, cette étude a mis en évidence, par-delà même les différences entre les processus décisionnels, que le concept d'autonomie, pourtant valorisé au plan législatif, joue un rôle peu important en Europe, les médecins gardant une culture "paternaliste» dans leurs pratiques de réanimation. On peut aussi citer l'enquête Eurled qui a fait le point sur le pratiques médicales en fin de vie dans 6 pays européens (Italie, Suède, Belgique, Danemark, Pays-Bas, Suisse) à partir des certificats de décès ${ }^{31}$ avec pour objectif de mesurer et comparer la fréquence des décisions médicales susceptibles d'abréger la vie. D'après l'enquête, un quart à la moitié des décès font l'objet d'une décision médicale relative à la décision de fin de vie, la plus grosse partie étant formée par des décisions de mise en œuvre de traitements de la douleur (avec pour effet d'abréger la vie). Ensuite, viennent les décisions de ne pas mettre en œuvre ou d'interrompre un traitement. Au total, le décès assisté est assez peu fréquent. L'étude démontre également que "même lorsque l'intention de mettre un terme à la vie du patient est explicite, celui-ci ou les membres de sa famille ne sont pas toujours impliqués dans le processus de décision ${ }^{32} \ldots$

\section{B. La consécration d'un véritable droit de choisir sa fin de vie}

\footnotetext{
${ }^{29} \S 62$ du rapport explicatif à la Convention.

${ }^{30}$ CDBI, 35 ième réunion, 2-5 décembre 2008, Les souhaits précédemment exprimés au sujets de soins de santé. Principes communs et différentes règles applicables dans les systèmes juridiques nationauxs, rapport préparé par le Professeur Roberto Andorno, http://www.coe.int/t/dg3/healthbioethic/Activities/09_Euthanasia/default_fr.asp

${ }^{31}$ J. Bilsen, J. Cohen et L. Deliens, La fin de vie en Europe : le point sur les pratiques médicales, Population et Sociétés, $\mathrm{n}^{\circ}$ 430, janvier 2007, pp. 1-4.

${ }^{32}$ J. Bilsen, J. Cohen et L. Deliens, La fin de vie en Europe : le point sur les pratiques médicales, op. cit., p. 3.
} 
La Cour européenne des droits de l'homme a joué, au travers de sa jurisprudence, un rôle important dans la consolidation de l'autonomie de l'individu en fin de vie. S'arrimant à l'un des articles de la Convention, l'article 8 relatif au droit au respect de la vie privée et familiale, le juge européen a en effet dégagé un véritable droit à l'autodétermination qui emporte, à la charge des Etats, de véritables obligations, néanmoins susceptibles, dans certaines conditions, de connaître certaines limites.

\section{1- L'apparition d'un droit à l'autodétermination}

L'on sait combien la notion de vie privée est une notion dont la Cour, loin de donner une définition exhaustive, a, au contraire, eu une acceptation très large afin d'assurer une protection forte de l'article 8. La Cour y a ainsi inclus, au-delà de la protection de la sphère intime de la personne, le droit au développement personnel mais encore le droit d'établir et d'entretenir des rapports avec d'autres êtres humains et le monde extérieur ${ }^{33}$. Dans son aspect "intime», la vie privée a également reçu une compréhension très étendue, recouvrant, on le sait, l'intégrité morale ou physique de la personne ${ }^{34}$, sa vie sexuelle ${ }^{35}$ ou encore son image ou ses données personnelles ${ }^{36}$. D'ailleurs, la Commission européenne des droits de l'homme, puis la Cour elle-même avaient déjà eu, à plusieurs reprises, l'occasion de laisser entendre que l'administration non consentie d'un traitement médical portait atteinte à l'article 8 et déclenchait la responsabilité de l'Etat partie pour violation de son obligation d'assurer une protection appropriée de l'intégrité physique de la personne. La Commission avait ainsi déjà affirmé qu'une "intervention médicale sous la contrainte, même si elle est d'importance minime, doit être considérée comme une atteinte à ce droit » ${ }^{37}$. La Cour avait emboité le pas estimant que l'administration de la diamorphine à un enfant de 12 ans que les médecins estimaient être entré en phase terminale, contre la volonté de la mère, "s'analyse en une atteinte au droit du premier au respect de sa vie privée et plus particulièrement à son droit à l'intégrité physique $»^{38}$ ou encore que l'administration forcée d'un traitement médical à une jeune femme majeure internée à la demande de son père dans un service de clinique psychiatrique porte atteinte au respect de la vie privée de l'intéressé39.

Il a toutefois fallu attendre l'arrêt Pretty du 29 avril 2002 pour avoir une confirmation de l'applicabilité de cette jurisprudence en situation de fin de vie. Faisant le

\footnotetext{
${ }^{33}$ CEDH, 16 décembre 1992, Niemetz cl Allemagne, Série A n 251-B, §29.

${ }^{34}$ CEDH, 26 mars 1985, X. et Y. cl Pays-Bas, Série A n ${ }^{\circ} 91, \S 21$ et s.

${ }^{35}$ Ibidem, $\S 22$.

${ }^{36} \mathrm{CEDH}, 21$ février 2002, Schüssel c/ Autriche, $\mathrm{n}^{\circ}$ 42409/98, non publiée.

${ }^{37}$ Com EDH, X. cl Autriche, 13 décembre 1979, DR 18, p. 154. Voir également, Com EDH, X. c/ Pays-Bas, 4 décembre 1978, DR 16, p. 86. Pour un autre exemple, dans une affaire Acmanne c/ Belgique du 10 décembre 1984, D.10435.83, la Commission avait admis le droit d'exercer un choix relatif à des traitements médicaux et avait analysé l'obligation de vaccination et d'examen radiologique comme des ingérences das l'exercice du droit au respect de la vie privée.

${ }^{38}$ CEDH, 9 mars 2004, Glass c/ Royaume Uni, Rec. 2004-II, §70.

${ }^{39}$ CEDH, 16 juin 2005, Storck c/ Allemagne, req $n^{\circ} 61603 / 00$, JCP G. 2005, I, 159, ${ }^{\circ}$ 15, chron. F. Sudre.
} 
constat qu'«à une époque où l'on assiste à une sophistication médicale croissante et à une augmentation de l'espérance de vie, de nombreuses personnes redoutent qu'on ne les force à se maintenir en vie jusqu'à un âge très avancé ou dans un état de grave délabrement physique ou mental aux antipodes de la perception aiguë qu'elles ont d'elles-mêmes et de leur identité personnelle », la Cour admet, sans difficulté, que la protection de l'intégrité de la personne âgée est garantie par l'article 8 et rappelle le principe précédent: "l'imposition d'un traitement médical sans le consentement du patient, s'il est adulte et sain d'esprit, s'analyserait en une atteinte à l'intégrité physique de l'intéressé pouvant mettre en cause les droits protégés par l'article $8 \$ b 1$ de la Convention $»^{40}$. Ici la différence est que Mme Pretty ne demandait pas à ne pas en bénéficier d'un traitement médical, mais souhaitait être assistée dans son suicide. La question était autrement plus délicate : il s'agissait de savoir si l'interdiction du suicide assistée tel que prévu par le droit anglais ${ }^{41}$ devait être considérée comme constituant une ingérence non autorisée dans le droit au respect de sa vie privée. Pour répondre à cette question, la Cour développe un raisonnement pour le moins audacieux qui débouche sur une innovation assez " considérable ${ }^{42}$. Bien qu'il n'ait été établi dans aucune affaire antérieure que l'article 8 de la Convention comporte un droit à l'autodétermination en tant que tel, la Cour considère ici que "la notion d'autonomie personnelle reflète un principe important qui sous-tend l'interprétation des garanties de l'article $8 »^{43}$ et peut s'entendre "au sens $d u$ droit d'opérer des choix concernant son propre corps ${ }^{44}$. La Cour affirme que, sur ce fondement, "la façon dont elle choisit de passer les derniers instants de son existence fait partie de l'acte de vivre et elle a le droit de demander que cela soit aussi respecté» et de conclure que "la requérante en l'espèce est empêchée par la loi d'exercer son choix d'éviter ce qui, à ses yeux, constituera une fin de vie indigne et pénible. La Cour ne peut exclure que cela représente une atteinte au droit de l'intéressé au respect de sa vie privée, au sens de l'article 8-1 de la Convention " ${ }^{45}$. La Cour confère au final à ce nouveau droit à l'autodétermination, depuis lors largement repris ${ }^{46}$, une portée très large puisque cela inclut le droit de choisir sa fin de vie, c'est-à-dire le droit de refuser de consentir à un traitement qui pourrait avoir pour effet de prolonger sa vie et plus généralement le droit de choisir le moment de sa mort et ses conditions.

\section{2- Les limites possibles au droit à l'autodétermination}

\footnotetext{
${ }^{40} \S 63$ de l'arrêt Pretty

${ }^{41}$ Cf note 18 .

${ }^{42}$ Olivier De Schutter, L'aide au suicide devant la Cour européenne des droits de l'homme, RTDH, 2003 , p. 84.

${ }^{43} \S 61$ de l'arrêt.

${ }^{44} \S 66$ de l'arrêt.

$45 \S 67$ de l'arrêt.

${ }^{46}$ Confirmation en est donnée par exemple par l'arrêt K.A et A. D. c/ Belgique du 17 février 2005 où la Cour consacre le droit de disposer de son corps et affirme que la notion d'autonomie personnelle peut s'entendre au sens d'opérer des choix concernant son propre corps (\$83). Le développement a d'ailleurs été tel qu'il a provoqué les réaction d'une partie de la doctrine qui souligne le paradoxe qu'il y aurait à déduire de l'autonomie personne la renonciation par une personne à son intégrité corporelle : Voir parmi d'autres Muriel Fabre-Magnan, Le domaine de l'autonomie personne, Indisponibilité du corps humain et justice sociale, Rec. Dalloz, 2008, p. 31 et $\mathrm{s}$.
} 
Toutefois, ce droit à l'autodétermination peut connaître des limites. Aux termes de l'article $8 \S 2$, les Etats ont en effet le droit de contrôler, au travers de l'application du droit pénal, les activités préjudiciables à la vie et à la sécurité d'autrui à condition que l'ingérence soit prévue par la loi et soit nécessaire, dans une société démocratique, à la poursuite d'un but légitime ${ }^{47}$. En l'espèce, ayant admis que l'interdiction faite à la requérante d'être assistée dans son suicide constituait une ingérence dans son droit au respect de sa vie privée, la Cour devait donc examiner l'éventuelle justification de cette ingérence au regard de l'article $8 \S 2$. Comme l'interdiction du suicide assisté était prévue par la loi et qu'elle poursuivait le but légitime de préserver la vie et donc les droits d'autrui, les difficultés se concentraient sur le point de savoir si l'ingérence était nécessaire, c'est-à-dire proportionnée au but légitime poursuivi. La Cour conclut que l'ingérence peut être considérée comme proportionnée étant donné " le risque d'abus et les conséquences probables des abus éventuellement commis qu'impliquerait un assouplissement de l'interdiction générale du suicide assisté ou la création d'exceptions au principe ${ }^{48}$, notamment pour les personnes les plus vulnérables. Elle construit son raisonnement, principalement ${ }^{49}$, autour de l'idée - peu rigoureuse juridiquement, mais plutôt convaincante empiriquement- de «pente glissante » ${ }^{50}$ : «en dépénalisant l'assistance au suicide dans certains cas déterminés d'où tout risque d'abus serait absent (...) l'on glisserait immanquablement vers une dépénalisation plus large, au détriment de la protection à laquelle ont droit de la part de l'Etat les personnes les plus vulnérables »51. C'est le risque de créer un précédent qui a fondé l'argumentation du juge européen. "Concrètement, elle révèle le souci légitime de la Cour de ne pas jouer aux apprentis sorciers en ouvrant trop facilement les portes de l'euthanasie aux personnes vulnérables à partir du cas exemplaire d'une requérante dont la lucidité et la saine détermination forçaient l'admiration ${ }^{52}$. Au total, si le droit de choisir le moment de sa mort et ses conditions afin d'éviter une fin de vie indigne semble incluse dans le droit au respect de la vie privée, en l'espèce la Cour juge que l'interdiction légale du suicide assisté s'avère nécessaire dans une société démocratique à la protection de la santé et de la sécurité publique. Ainsi semble s'esquisser une différence entre le droit de choisir sa fin de vie et les conditions de sa mort et le droit de mettre fin à ses jours.

\footnotetext{
${ }^{47}$ Selon la disposition, il s'agit de des mesures nécessaires à «la sécurité nationale, à la sûreté publique, au bien-être économique du pays, à la défense de l'ordre et à la prévention des infractions pénales, à la protection de la santé ou de la morale, ou à la protection des droits et libertés d'autrui ».

48 § 74 de l'arrêt.

${ }^{49}$ Plus étonnants sont les propos aditionnels de la Cour qui, pour conclure à la proportionnalité de la loi anglaise, rappelle que les poursuites pénales qu'elles prévoient pour les proches qui assistent un malade à se suicider ne sont pas toujours mises à exécution! L'absence de violation d'un droit fondamental de la Convention dépend ici des possibilités de non application de la loi... : voir le $\S 76$ où la Cour explique qu'il ne lui paraît pas arbitraire que «le droit reflète l'importance du droit à la vie en interdisant le suicide assisté tout en prévoyant un régime d'application et d'appréciation par la justice qui permet de prendre en compte dans chaque cas concret tant l'intérêt public à entamer des poursuites que les exigences justes et adéquates de la rétribution et de la dissuasion ».

${ }^{50}$ Olivier De Schutter, L'aide au suicide devant la Cour européenne des droits de l'homme, op. cit., p. 92.

${ }^{51}$ Surtout si l'on se souvient que, médicalement, toute personne en phase terminale d'une maladie est le plus souvent gravement déprimée et donc de ce fait vulnérable.

52 Jean-Pierre Marguénaud, Hymnes à la vie et à l'autonomie personnelle, op. cit., p. 859.
} 


\section{II- DECIDER DE MOURIR}

La décision de mourir qui renvoie au point le plus extrême de la fin de la vie est au centre de débats passionnés entre les partisans d'une autonomie complète étendue jusqu'à la possibilité de choisir la mort et les défenseurs de l'interdit de la mort "donnée », quelque soit les raisons. Logiquement et heureusement pourrait-on dire, dans ce contexte, le droit européen a emprunté nettement la voie de la discrétion. Précisant que le droit à la vie n'implique en aucun cas un droit de mourir (A), le droit européen est en effet resté très prudent dans le débat sur l'euthanasie (B).

\section{A. Le refus du juge européen de consacrer un droit de mourir}

Le refus du juge européen est tout à la fois net et logique.

1- La netteté de la position du juge européen

L'arrêt Pretty présentait un second intérêt en ce que la requérante invoquait l'article 2 qui dispose que «le droit de toute personne à la vie est protégée par la loi ». La requérante soutenait que l'autoriser à se faire aider pour mettre un terme à son existence ne serait pas contraire à cet article au motif que ce dernier garantirait non seulement le droit à la vie, mais également, de façon corollaire, le droit de mourir. Il faut dire que certains droits garantis par la Convention ont été interprétés comme conférant des droits à ne pas faire, c'est-à-dire des droits constituant l'antithèse de ce que le droit explicitement reconnu autorise à faire. L'article 11 par exemple relatif à la liberté d'association confère, selon la Cour, un droit à ne pas adhérer à une association ${ }^{53}$; l'article 9 sur la liberté de conscience et de religion comporte un droit à ne pas être soumis à aucune obligation d'exprimer ses pensées, de changer d'avis ou de divulguer ses convictions ${ }^{54}$. La réponse de la Cour est, à propos de l'article 2, nette. Explicitant bien la spécificité du droit à la vie, qui à la différence des libertés "n'implique aucune dimension de "choix» dans le chef des individus » ${ }^{55}$, elle en conclu que l'article 2 «ne saurait, sans distorsion de langage, être interprété comme conférant un droit diamétralement opposé, à savoir un droit à mourir ; il ne saurait davantage créer un droit à l'autodétermination au sens où il donnerait à l'individu le droit de choisir la mort plutôt que la vie (...) La Cour estime donc qu'il n'est pas possible de déduire de l'article 2 de la Convention un droit à mourir, que ce soit de la main d'un tiers ou avec l'assistance d'une autorité publique ${ }^{56}$. Ce refus de reconnaître a droit à la vie un aspect négatif peut être "critiquées, discutées, regrettées, force est néanmoins de reconnaître qu'elles tranchent une question de principe avec courage et netteté sans ensevelir la réponse, comme il arrive

\footnotetext{
${ }^{53}$ Voir par exemple CEDH, 29 avril 1999, Chassagnou et al. c/ France, n 25088/94, 28331/95 et 28443/95, $\S \S$ 91-92, CEDH 1999-III, \$103.

${ }^{54}$ CEDH, 23 octobre 1990, Darby, A. 187

${ }^{55}$ Olivier De Schutter, L'aide au suicide devant la Cour européenne des droits de l'homme, op. cit., p. 79.

${ }^{56} \S 39-40$ de l'arrêt.
} 
trop souvent, sous un fatras méthodologique dérivant du concept particulièrement flou de « marge nationale d'appréciation » »57

\section{2- La logique de la position du juge européen}

Cette affirmation très forte est parfaitement cohérente avec l'obligation positive que le juge européen met, depuis longtemps, à la charge des Etats au titre de l'article 2, celle non seulement de s'abstenir de donner la mort de manière intentionnelle, mais aussi de prendre les mesures nécessaires à la protection de vie ${ }^{58}$. Elle l'est également avec la valeur reconnue par la Cour à l'article 2. Le droit à la vie est, de jurisprudence constante, considéré comme «l'une des valeurs fondamentales des sociétés démocratiques qui forment le Conseil de l'Europe » ${ }^{59}$. D'ailleurs, l'arrêt accentue encore la «valorisation» de ce droit puisqu'elle va jusqu'à lui accorder ici «la prééminence » au motif que, sans lui, « la jouissance de l'un quelconque des autres droits et libertés garantis par la Convention serait illusoire» ${ }^{60}$. "La Cour a ici réalisé une avancée significative dans l'établissement de la hiérarchie des droits de l'homme en désignant clairement «le Roi des Droits » ${ }^{61}$.

\section{B. L'abstention du droit européen dans le débat sur l'euthanasie}

Il est classique de distinguer entre l'euthanasie passive où la mort n'est pas l'effet directement recherché, mais ce qui l'est c'est l'administration ou le refus de soins qui auront pour effet secondaire d'entraîner la mort et l'euthanasie active où la mort est l'effet recherché. C'est cette seconde forme d'euthanasie, qui recouvre le suicide assistée, mais également toutes décisions, notamment médicales ayant pour but directement de mettre un terme à une vie, qui fait débat. Naturellement, ce débat se retrouve au plan européen où la tension est perceptible entre les instances qui prônent l'autonomie des malades au stade terminal ${ }^{62}$ et celles qui réaffirment l'interdit de tuer ${ }^{63}$. Faute de consensus, pour l'heure, la situation est pour le moins paradoxale : l'euthanasie est en effet, dans la majorité des pays européens, un délit, mais se pratique tous les jours. Seules les lois belges ${ }^{64}$ et néerlandaises ${ }^{65}$ entrées en vigueur en 2002 permettent aux

\footnotetext{
${ }^{57}$ Jean-Pierre Marguénaud, Hymnes à la vie et à l'autonomie personnelle, op.cit., p. 858.

${ }^{58}$ CEDH, 9 juin 1998, L.C.B. c/ Royaume-Uni, Rec. 1998-III . p. 1403, § 36.

${ }^{59} \mathrm{CEDH}, 27$ septembre 1995, Mac Cann c/ Royaume Uni, série A, n 324, §147.

${ }^{60} \S 37$ de l'arrêt.

${ }^{61}$ Jean-Pierre Marguénaud, Hymnes à la vie et à l'autonomie personnelle, op. cit., p. 858.

${ }^{62}$ Proposition de résolution sur l'assistance aux mourants, adoptée par le Parlement européen le 25 avril 1991 , article 8. 43.

${ }^{63}$ Recommandation $n^{\circ} 1418$ du Conseil de l'Europe du 25 juin 1999 sur la protection des droits de l'homme et la dignité des malades mourants ou en phase terminale, approuvée le 26 mars 2002, $\$ 9$.

${ }^{64}$ On entend par « la loi belge » la loi du 28 mai 2002 relative à l'euthanasie (Mon. b., 22 juin 2002 , http://www.just.fgov.be/cgi/welcome.pl) Le médecin qui pratique une euthanasie ne commet pas d'infraction s'il respecte les conditions et procédures prescrites et il s'est assuré que :

- le patient est majeur ou mineur émancipé, capable et conscient au moment de sa demande (qui doit être faite par écrit) ;

- la demande est formulée de manière volontaire, réfléchie et répétée, et qu'elle ne résulte pas d'une pression extérieure ;
} 
médecins qui accèdent à des demandes d'euthanasie volontaire active ou de suicide assisté formulée par un patient d'échapper aux poursuites, dans certaines conditions rigoureuses ${ }^{66}$.

Or, à son propos, sauf une exception mais d'une valeur uniquement recommandatoire ${ }^{67}$, le droit européen reste particulièrement peu $\operatorname{disert}^{68}$ : à l'impossible consensus textuel s'ajoute la discrétion du juge européen en ce domaine.

\section{1- L'absence de consensus textuel}

Dans la Convention d'Oveido, dès le départ, il avait été décidé de ne pas aborder deux problèmes éthiques importants : l'avortement et l'euthanasie. Vu les divergences d'opinions dans les Etats membres et entre les Etats membres, aucun accord fructueux sur ces deux questions ne pouvait être escompté. En 2001, une enquête menée par le CDBI à la demande du Comité des Ministres avait d'ailleurs confirmé l'hétérogénéité des lois et pratiques pertinentes en matière d'euthanasie et autres décisions sur la fin de

- le patient se trouve dans une situation médicale sans issue et fait état d'une souffrance physique ou psychique constante et insupportable qui ne peut être apaisée et qui résulte d'une affection accidentelle ou pathologique grave et incurable.

${ }^{65} \mathrm{La}$ loi relative au «contrôle des interruptions de vie pratiquées à la demande du patient et des aides au suicide », entrée en vigueur aux Pays-Bas le $1^{\mathrm{e}}$ avril 2002. En substance, la nouvelle loi contient un amendement à l'article 293 du Code pénal stipulant que, bien que quiconque mettant fin à la vie d'une personne sur demande expresse et ardente de ladite personne soit passible d'une peine d'emprisonnement ne dépassant pas douze ans ou d'une

amende de cinquième catégorie, un tel acte ne sera pas un délit s'il est perpétré par un médecin qui en notifie l'expert médico-légal de la commune conformément à la législation en vigueur et qui remplit les critères de soin stipulés. Selon ces critères, le médecin concerné doit :

a. avoir la conviction que le malade a fait une demande volontaire et mûrement réfléchie ;

b. avoir la conviction que la souffrance du malade est intolérable et qu'il n'y a aucune perspective d'amélioration ;

c. avoir informé le malade sur sa situation et ses perspectives ;

d. être arrivé à la conclusion, avec le malade, qu'il n'y a pas d'alternative possible compte tenu de sa situation ;

e. avoir consulté au moins un autre médecin indépendant, lequel doit avoir vu le malade et donné un avis écrit sur les critères de soin énoncés plus haut (voir les paragraphes a. à d.) ;

f. avoir mis fin à la vie du malade ou aidé à son suicide avec tous les soins médicaux et l'attention qui se doivent.

${ }^{66}$ Yves-Henri Leleu et Gilles Genicot, L'euthanasie en Belgique et aux Pays-Bas, Variations sur le thème de l'autodétermination, RTDH, 2004, n $^{\circ}$ 57, pp. 5-50.

${ }^{67}$ La Recommandation 1418 (1999) de l'Asemblée parlementaire du Conseil de l'Europe énonce au paragraphe 9: «L'Assemblée recommande par conséquent au Comité des Ministres d'encourager les Etats membres du Conseil de l'Europe à respecter et à protéger la dignité des malades incurables et des mourants à tous égards: c. en maintenant l'interdiction absolue de mettre intentionnellement fin à la vie des malades incurables et des mourants:

$i$. vu que le droit à la vie, notamment en ce qui concerne les malades incurables et les mourants, est garanti par les Etats membres, conformément à l'article 2 de la Convention européenne des Droits de l'Homme qui dispose que "la mort ne peut être infligée à quiconque intentionnellement »;

ii. vu que le désir de mourir exprimé par un malade incurable ou un mourant ne peut jamais constituer un fondement juridique à sa mort de la main d'un tiers;

iii. vu que le désir de mourir exprimé par un malade incurable ou un mourant ne peut en soi servir de justification légale à l'exécution d'actions destinées à entraîner la mort ».

${ }^{68}$ Voir sur ce sujet : Christian Byk, Euthanasie : la nécessité d'une loi au regard du droit européen des droits de l'homme? disponible sur le site : www.iales.org/dossiers/.../euthanasie-la-necessite-d-une-loi.pdf 
vie $^{69}$. Un peu plus tard, la Commission des questions sociales, de la santé et de la famille de l'Assemblée parlementaire rendait, quatre ans après l'adoption de la Recommandation 1418, un rapport, le Rapport Marty, le 10 septembre 2003 qui préconisait l'adoption d'une Résolution sur ce sujet. Toutefois, faute de majorité, cette résolution n'a jamais été adoptée.

\section{2- La discrétion du juge européen}

Surtout, le juge européen est resté très discret sur cette question. Certes, dans son arrêt Pretty, il a conclu à l'absence d'incompatibilité d'une interdiction générale (et d'une incrimination) du suicide assisté, telle que celle prévue par la loi anglaise, avec les articles $2,3^{70}$ et 8 de la Convention. Toutefois, s'il y a un risque que l'arrêt Pretty soit lu de manière à lui faire dire ce qu'il ne dit pas, c'est-à-dire comme une condamnation implicite de la dépénalisation de l'euthanasie ${ }^{71}$, il faut bien admettre que l'arrêt ne dit rien de tel. La Cour souligne que le refus de toute dépénalisation ne constitue pas une violation de l'article 2 si certaines justifications sont présentes, mais elle ne dit rien sur l'admission d'une dépénalisation $d u$ suicide assisté ou plus généralement de l'euthanasie. La Cour aurait eu l'occasion de s'exprimer à nouveau sur la question de l'euthanasie dans l'affaire relative à Eluana Englaro ${ }^{72}$ déclenchée par la décision de la Cour d'appel de Milan autorisant le père d'Eluana à faire interrompre l'alimentation et

\footnotetext{
${ }^{69}$ Le CDBI avait adressé aux Etats membres du Conseil de l'Europe un questionnaire relatif à leurs lois et pratiques en matière d'euthanasie et autres décisions sur la fin de vie. Le document final contient une analyse des réponses des 35 Etats membres ayant répondu. L'expert qui a dirigé l'enquête a également rédigé un rapport concomitant, non publié par le CDBI. Rapport sur les lois en vigueur ou pratiques appliquées dans les Etats membres relatives aux questions soulevées par la Recommandation 1418 (1999) de l'Assemblée parlementaire relative à la protection des droits de l'homme et de la dignité des malades incurables et des mourants [831 Réunion] : Réponses au questionnaire pour les Etats membres relatif à l'euthanasie, 20 janvier 2003, CDBI/INF (2003) 8

${ }^{70}$ L'incrimination de l'assistance au suicide n'est pas un traitement inhumain ou dégradant selon la Cour : «en l'espèce, la souffrance de la requérante n'est pas la conséquence d'un comportement actif des autorités. Ce n'est pas plus la conséquence d'un défaut de protection vis-à-vis des agissements d'un tiers. Ce n'est pas non plus l'inaction des autorités, leur défaut de prendre en compte la maladie d'une personne dont elles ont la charge qui sont en cause. La souffrance, aussi grande soit-elle, résultant de la maladie ne peut être considérée comme un traitement ». Florence Massias, Note sous l'arrêt, Revue de science criminelle, 2002, p. 647. En bref, personne ne pouvait sérieusement reprocher à l'Etat d'avoir infligé à la malade le moindre mauvais traitement puisqu'elle avait toujours reçu les soins adéquats de la part des autorités médicales.

${ }^{71}$ Olivier De Schutter, L'aide au suicide devant la Cour européenne des droits de l'homme, op. cit., p. 74.

${ }^{72}$ Victime d'un accident de la route survenu en janvier 1992, au cours duquel elle subit un traumatisme crânien et se fractura une vertèbre, une jeune femme âgée de 20 ans prénommée E.E. tomba dans le coma. Par la suite, on diagnostiqua chez elle un état végétatif avec tétraplégie spastique et perte de toute faculté psychique supérieure.
}

En décembre 1996, E.E. fut placée sous la tutelle de son père. En janvier 1999, celui-ci diligenta une procédure en vue d'obtenir l'autorisation de faire interrompre l'alimentation et l'hydratation artificielles de sa fille, soutenant que tel aurait été le vœu de celle-ci compte tenu de sa personnalité et des idées qu'elle avait exprimées sur la vie et la dignité humaine avant son accident. Refusée à deux reprises, l'autorisation est finalement donnée par une décision du 25 juin 2008, de la cour d'appel de Milan, statuant sur renvoi, au double motif que l'état végétatif de celle-ci était irréversible et qu'il existait des preuves claires, concordantes et convaincantes de ce que cette demande reflétait fidèlement la volonté de la personne représentée telle qu'elle pouvait se déduire du style de vie de celle-ci, de ses convictions et des vues qu'elle avait exprimées sur la dignité humaine avant de sombrer dans l'inconscience. 
l'hydratation artificielle de sa fille. En effet, dans l'arrêt Ada Rossi et autres \& sept requêtes $c$. Italie ${ }^{73}$, plusieurs tuteurs de personnes en état végétatif, quelques associations composées notamment de proches de personnes lourdement handicapées, de médecins, de psychologues et d'avocats assistant ces personnes ainsi qu'une association de défense des droits de l'homme avaient introduit une requête devant la Cour se plaignant des effets négatifs que l'exécution de l'arrêt rendu par la Cour d'appel de Milan pourrait avoir à leur égard, notamment en ce qu'elle engendrerait une discrimination pour les personnes lourdement handicapées et les mettrait à la merci des tiers qui pourraient librement disposer de leur vie. Toutefois, la Cour n'a pas eu à se prononcer sur le fond, déclarant les requêtes irrecevables au motif que les requérants ne pouvaient être considérés comme victimes d'une violation de la Convention aux sens de l'article 34 de la Convention ${ }^{74}$. Pour l'heure, donc cette question n'a jamais été soumise directement au jugement de la Cour.

Il pourrait d'ailleurs tout à fait être soutenu que la dépénalisation de l'euthanasie n'équivaudrait pas à la reconnaissance d'un droit à mettre fin à sa vie et pourrait donc être compatible avec l'article 2 , sous certaines conditions précises, en particulier que le malade en ait fait la demande consciente ${ }^{75}$. Le Conseil d'Etat belge, dans son avis sur la loi alors en préparation avait abouti à une telle conclusion ${ }^{76}$. Il avait noté, en particulier, après analyse de la jurisprudence de la Cour européenne des droits de l'homme, que l'obligation positive qu'ont les Parties de protéger le droit à la vie ne doit pas empêcher de tenir compte, notamment, du droit des personnes à l'autodétermination. Cela signifie que l'obligation des autorités de protéger le droit à la vie doit être placée en regard du droit des personnes à être protégées des peines ou traitements inhumains et du droit à l'intégrité physique et morale, qui dérive du droit au respect de la vie privée. Or, la Convention ne donne pas de directive sur la manière de résoudre ce conflit entre les

\footnotetext{
${ }^{73}$ Deuxième Section, 16 décembre 2008, requête $n^{\circ} 55185 / 08$ et autres.

${ }^{74}$ La Cour rappelle que cet article « exige qu'un individu requérant se prétende effectivement lésé par la violation qu'il allègue. Il n'institue pas au profit des particuliers une sorte d'actio popularis pour l'interprétation de la Convention; il ne les autorise pas à se plaindre in abstracto d'une loi par cela seul qu'elle leur semble enfreindre la Convention. En principe, il ne suffit pas à un individu requérant de soutenir qu'une loi viole par sa simple existence les droits dont il jouit aux termes de la Convention ; elle doit avoir été appliquée à son détriment. Toutefois, la Cour a déjà admis qu'un requérant peut se prétendre potentiellement victime d'une violation de la Convention lorsqu'il n'est pas en mesure de démontrer que la législation incriminée lui a été effectivement appliquée, pour autant qu'il existe des indices raisonnables et convaincants de la probabilité de réalisation d'une violation en ce qui le concerne personnellement. En l'espèce, la Cour estime les requérants n'ont pas satisfait à cette obligation. D'abord, la juridiction s'est prononcée dans des circonstances bien précises, ensuite, elle a bien rappelé l'indispensable prise en compte de la volonté du malade exprimée par le tuteur.

${ }^{75}$ En outre, il est impératif que l'état médical soit sans issue et constaté comme tel. La question est évidemment plus délicate pour les personnes inconscientes ou incapables de manifester leur volonté d'où l'intérêt d'une déclaration anticipée. On comprend dès lors que les lois belge et néerlandaise prévoit que l'élément fondamental qui permet de justifier le mécanisme est la demande du patient, fût-elle anticipée. Les conditions relatives à la qualité de la demande font obstacle à ce que celle-ci émane de personnes inconscientes (coma, état végétatif permanent...) ou ne disposant plus d'une clarté d'esprit suffisante pour la formuler (maladie d'Alzheimer). Les deux législateurs leur permettent néanmoins de bénéficier d'une euthanasie grâce à la technique de la déclaration anticipée.

${ }^{76}$ Conseil d'Etat, section de législation, avis du 2 juillet 2001, Doc. parl., Sénat, 2000-2001, no 2-244/21, Rev. trim. dr. h., 2001, p. 259, note F. Rigaux.
} 
droits fondamentaux. Selon la haute Assemblée, il revient d'abord au législateur d'user de son pouvoir discrétionnaire pour résoudre ce conflit, le juge (et l'interprète) devant « respecter le pouvoir d'appréciation du législateur et ne pouvant se substituer à lui ». La Cour a déjà tenu ce genre de propos, affirmant ainsi que «le législateur étant le mieux à même d'arbitrer entre les divers courants politiques et éthiques, on comprend qu'il faille s'interdire de juger son ouvre d'un point de vue moral, du moins en tant que juriste et respecter son choix de faire prévaloir la liberté individuelle contre l'opinion selon laquelle la vie humaine est rigoureusement et radicalement indisponible »77. Quelque soit l'interprétation, en tous les cas, dans le débat sur la dépénalisation de l'euthanasie, la jurisprudence européenne «laisse ouverte la voie de l'expérimentation »78.

Au final, le droit européen n'apporte, sur les questions relatives à la fin de vie, que des « morceaux » de réponses, quelques jalons ici et là (absence du droit de mourir, mais existence d'un droit de choisir sa mort) qui encadrent les choix des Etats certes, mais bien à la marge. N'est-ce toutefois par ici «faire preuve de sagesse face à la diversité des conceptions sur ce qu'implique le respect de la dignité de la personne humaine en fin de vie ${ }^{79}$ ? En d'autres termes, n'est-ce pas heureux que le droit européen n'éclaire pas trop la nuit qu'est notre mort ...?

Biographie :

Maître de conférences en droit public à l'Université Aix-Marseille III (France), Estelle Brosset est spécialisée en droit de l'Union européenne, droit de l'environnement et droit de la santé. Elle a dirigé plusieurs ouvrages collectifs en la matière, en particulier un ouvrage sur le droit international et européen du vivant (la Documentation française, 2009) et un ouvrage sur les enjeux de la normalisation technique internationale dans les domaines environnementales et sanitaires (la Documentation française, 2006). Elle est charge de plusieurs enseignements dans les Masters 2 de droit de la santé et le Master de droit international et européen de l'environnement de la Faculté de droit d'Aix-enProvence.

\footnotetext{
${ }^{77}$ CEDH, 19 mai 1982, H. c. Norvège.

${ }^{78}$ Olivier De Schutter, L'aide au suicide devant la Cour européenne des droits de l'homme, op. cit., p. 111.

${ }^{79}$ Louis Dubouis, La fin de vie et le droit européen op. cit., p. 87.
} 